16 May 2011

\title{
Perception of relative depth interval: systematic biases in perceived depth
}

\author{
Harris $^{1, *}$, J.M., Chopin ${ }^{1,2}$, A., Zeiner ${ }^{1}$, K.M., Hibbard ${ }^{1}$, P.B.
}

Quarterly Journal of Experimental Psychology: IN PRESS

Please do not cite or quote without our permission.

1: Vision Lab, School of Psychology, University of St. Andrews, Scotland, UK.

2: Laboratoire Psychologie de la Perception, Université Paris Descartes \& CNRS, France

* Corresponding author. Vision Lab, School of Psychology, University of St. Andrews, South

St., St. Andrews, KY16 9JP, Scotland, UK. Tel: +44-1334-462061. Fax: +44-1334-463042.

email: julie.harris@st-andrews.ac.uk

Short title: Biases in depth interval perception 


\section{Abstract}

Given an estimate of the binocular disparity between a pair of points, and an estimate of the viewing distance, or knowledge of eye position, it should be possible to obtain an estimate of their depth separation. Here we show that, when points are arranged in different vertical geometric configurations across two intervals, many observers find this task difficult. Those who can do the task, tend to perceive the depth interval in one configuration as very different from depth in the other configuration. We explore two plausible explanations for this effect. The first is the tilt of the empirical vertical horopter: points perceived along an apparently vertical line correspond to a physical line of points tilted backwards in space. Second, the eyes can rotate in response to a particular stimulus. Without compensation for this rotation, biases in depth perception would result. We measured cyclovergence indirectly, using a standard psychophysical task, while observers viewed our depth configuration. Biases predicted from error due either to cyclovergence, or the tilted vertical horopter, were not consistent with the depth configuration results. Our data suggest that, even for the simplest scenes, we do not have ready access to metric depth from binocular disparity.

Keywords: binocular disparity, depth, depth interval, metric depth, cyclovergence, vertical horopter. 


\section{Introduction}

The tiny angular differences between the right and left eyes' views of a scene are used by the brain to extract relative depth (see Howard, 2002). These arise because the two eyes view the world from slightly different vantage points. The image locations of a point in the world on each eye's retina depend on its position in space, and these locations may be the same or different in the two eyes. For the images to fall at corresponding locations in the two eyes, a point must lie on the locus of points in space known as the horopter, defined by the orientations of the eyes. More typically, points will project to non-corresponding locations in the two eyes. The differences in location, or binocular disparities, are a powerful cue to depth. Whilst we are exquisitely sensitive to even tiny differences in binocular disparity between points, the visual system cannot simply 'read-out' the disparity at every point in the world so as to deliver a depth-map of the scene. More information than simply an estimate of this disparity is needed before information about metric depth (depth in an external-world unit, such as $\mathrm{cm}$ ) can be extracted.

Human sensitivity to binocular disparity has been much studied (e.g. Howard, 2002), but most tasks involving disparity detection have not been designed to address how we use disparity to estimate depth. Typically, one would show observers a pair of intervals, each containing a pair of dots at different disparities (figure 1a), and ask in which the depth separation is larger. It is well known that observers can perform this task with reasonable precision for very simple stimuli. However, observers become less precise at judging differences between depth separations as the depth separation becomes larger (e.g. Ogle, 1958, Blakemore, 1970). For example, using a depth pedestal of around $20 \mathrm{~min}$ arc, discrimination thresholds are around 1 min arc disparity, an order of magnitude larger than for a zero depth pedestal (Blakemore, 1970; Badcock \& Schor, 1985). When asked to make even simple ordinal depth judgments for 
points on more complex, rendered objects, precision is worse than for isolated pairs of points (Norman \& Todd, 1998).

(a)

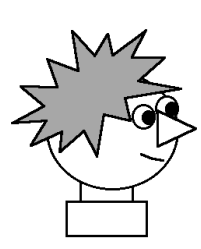

standard

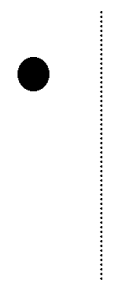

0

(b)

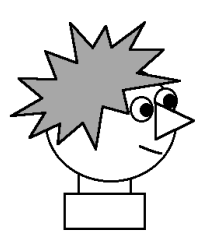

standard

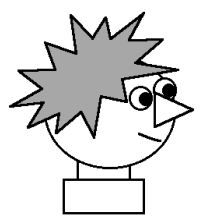

test

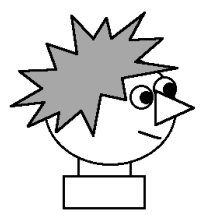

test
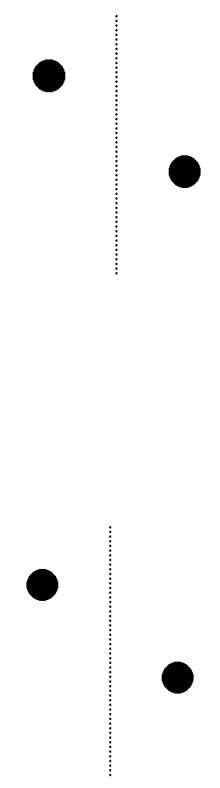

\section{Figure 1}

(a) 'Same' configuration. Cartoon showing a side-on view of the observer viewing the standard and test intervals. Note that the configuration (top-near) is the same for both intervals. (b) 'Different' configuration. Here the standard interval displays the top-far configuration and the test interval the top-near configuration.

However, this basic task does not address possible systematic errors in the extraction of depth from binocular disparity. Errors are possible because, in order to obtain depth from disparity, the direction in which each eye is pointing must be known. Changes in the viewing geometry can affect both the location of the image of a point in one eye, and its binocular disparity. For example, consider converging the eyes so that they are both looking at the same point.

Changing convergence to look at a nearer or further point will not change the relative binocular disparity between the points, but will change the location of the points on each retina. 
Cyclotorsion, or rotation of one eye around the visual axis, can affect the disparity of all points in the image. Thus, if perfect knowledge of the viewing geometry is not available, this will introduce errors in the perceived three-dimensional location of points. For example, it is well established that underestimating the distance to an object (equivalent to overestimating the vergence angle when it is fixated) will lead to an underestimation of the depth difference between points inferred from their binocular disparity (Johnston, 1991; Brenner and Landy, 1999; Scarfe and Hibbard, 2006).

Our aim here was to devise an experiment to test for the potential influence of ignorance of the viewing geometry. To do this, one should use a task and stimulus that require correct disparity recovery and knowledge of viewing geometry. There are many studies that have looked at the issue of needing to know viewing geometry. We wanted to specifically study this issue in the context of simple depth discrimination. Here, we chose a task where the observer estimates a difference in depth separation between pairs of points, shown in two different time intervals. Figure $1 \mathrm{~b}$ shows an experimental situation in which an observer is shown two intervals, each containing pairs of dots with a depth separation. The disparity between them is the same as in figure 1a, the difference is the configuration of the points (whether the upper one is closer or farther) between the two intervals. Comparing depth interval judgments from stimuli where both intervals had the same depth configuration (figure 1a), with judgments where the intervals had a different configuration (figure 1b), allowed us to explore the extent to which judgements are affected by errors occurring at the disparity recovery stage, or errors due to imperfect compensation for viewing geometry at later stages linked to depth extraction.

We will explore two ways in which we expect the visual system to make errors. Mathematical predictions for the effects of each source of bias are detailed in the Appendix. First, comparing perceived depth for different configurations allows us to test for disparity recovery errors. When 
the foveae and the horizontal meridians of the eyes are aligned, points that fall on corresponding locations in the two eyes are defined as having zero binocular disparity and are said to fall along the geometrical horopter. The geometric horopter is defined as the locus of points in space that project to corresponding points in the two eyes: the locus of zero binocular disparity. Points falling off the geometric horopter have a non-zero binocular disparity. In the horizontal plane, for close viewing, the horizontal geometrical horopter (also called the ViethMuller circle) is a circle passing through the nodal points of the two eyes and the fixation point. The vertical geometrical horopter is a vertical line lying in the head's midsagittal plane and intersects the Vieth-Muller circle. The empirical horopter is defined as the locus of points that appear to lie in the same direction from each eye. It is known that the geometric and empirical horopters are different. The empirical horizontal horopter tends to be less concave than the Vieth-Muller circle, while the empirical vertical horopter is a line, tilted backwards, in such a way that it passes through the fixation point, and a point near the feet (e.g. Nakayama, 1977; Ledgway \& Rogers, 1999; Schreiber et al, 2008). This means that points that lie along a line tilted slightly back from vertical appear to be vertical. This difference between the geometric and empirical horopters could potentially introduce errors in determining the distance of points from the observer. If depth judgments were made relative to this tilted vertical horopter, different depths might be perceived for stimuli tilted top-far (figure $1 \mathrm{~b}$, left), as opposed to topnear (figure 1b, right). More specifically, with a backward tilted vertical horopter, one would expect greater perceived depth in the top-near condition, which would contain a greater effective disparity relative to the empirical horopter than the top-far condition (see figure 8 in Appendix). There is some evidence for anisotropy in depth perception for upper and lower visual fields, which would be consistent with error due to a backward tilted horopter. Breitmeyer, Julesz \& Kropfl (1975) found that near patches were detected more rapidly than far patches in the lower visual field, confirmed by Previc et al (1995). However, other labs have failed to find an anisotropy (Manning et al, 1987). 
Second, the depth obtained could also depend on the attitude and orientation of the eyes. We know that the eyes can undergo rotational, torsional movements in opposite directions around the visual axis (cyclovergence) in response to stimuli that rotate in opposite directions in the two eyes' views (e.g. reviewed by Maxwell \& Schor, 2006). Whilst the eyes seem to exhibit very few spontaneous changes in cyclovergence, some observers do show a rotational offset (a cyclophoria) when fixating a single point, as opposed to a patterned background (van Rijn, van der Steen, \& Collewijn, 1992). If the brain could compensate for the cyclovergence by combining retinal and extra-retinal information (as it can to some extent for versional eye movements, synchronous movement of both eyes, e.g. Freeman, 2001, Perrone \& Krauzlis, 2008; and horizontal vergence changes, e.g. Nefs \& Harris, 2007, 2008), the rotational state of the eyes would not affect the perception of depth. But there is evidence that such rotational compensation does not take place (van Ee \& van Dam, 2003). Without compensation, persistent misalignments of the eyes could result in biases in depth perception (see figure 7 in Appendix). Recent work has suggested that errors in cyclovergence might account for errors in perceived depth in curved line stereograms (Mitsudo, 2007), and this has been supported by work showing that perceived depth in such displays can be altered by inducing additional cyclovergence (Mitsudo, Kaneko \& Nishida, 2009).

We explored here whether precision and bias in perceived depth could be linked to the tilt of the vertical horopter or small, individual-specific offsets in cyclovergence. We measured depth discrimination for the depth separation between a pair of points, for two different configurations, specifically testing for biases in the direction predicted by the tilted vertical horopter. In the same experiment, we measured cyclovergence, to test for the existence of biases linked to small mis-rotations of the eyes. 


\section{Methods}

\section{Observers}

Twenty four observers were recruited, with ages ranging between 18 and 38 . Four of these were experienced psychophysical observers (CG, HN, AC, VD), the others completely naïve to the purposes of the experiment, with most recruited by an online subject recruitment system. We pre-screened observers for stereoacuity within the normal range, using the TNO stereotest. All observers reported normal or corrected-to-normal vision and gave written consent for their participation. The inexperienced group were paid expenses for their participation. The University Teaching and Research Ethics Committee (UTREC) of the University of St. Andrews approved all experiments.

\section{Apparatus}

Visual stimuli were displayed on an liyama 22in Vision-Master-Pro CRT monitor with a resolution of $1280 \times 1024$ pixels and a refresh rate of $100 \mathrm{~Hz}$. Stimulus generation and display was achieved via Matlab and the Psychophysics Toolbox (Brainard, 1997; Pelli, 1997), using a standard pc workstation. Stimuli were presented side by side on a single monitor and a modified Wheatstone stereoscope was used to deliver a single stimulus to each eye, at a viewing distance of $1 \mathrm{~m}$. Observers were seated in a darkened room and their position was stabilised via a chin-rest.

\section{Stimuli and procedure}

In a single experiment, we presented observers with two different types of stimulus, and asked them to perform two tasks at the end of every trial. Task 1 involved judging which of two intervals contained the pair of points with the larger depth separation. Points were bright dots

$\left(62 \mathrm{~cd} \mathrm{~m}^{-2}\right)$ on a dark background (luminance effectively $0 \mathrm{~cd} \mathrm{~m}^{-2}$, below the limit of our Minolta100 luminance meter), of diameter $0.28 \mathrm{~cm}$ (about $1 \mathrm{~min}$ arc), presented one above the other, 
with a height separation of $5 \mathrm{~cm}$ (2.86 deg). There was a small horizontal offset applied to each of the top and bottom point, at random (range $+-0.5 \mathrm{~cm}$ ) but in both eyes' views (no additional binocular disparity), so that the task could not be completed on the basis of information in a single eye's image. Observers viewed a dark screen, in the centre of which was a fixation cross ( 2 by $2 \mathrm{~cm}$ ), and initiated a trial using a button press. The first stimulus interval lasted for two seconds, followed by a one second blank inter-stimulus interval (in which the fixation cross was presented), followed by the second stimulus interval, also two seconds (see figure 2). The stimuli in these two intervals could have either the same configuration (top-near, top-near, as in figure 1a, or top-far, top-far) or the different configuration (top-far, top-near, as in figure $1 \mathrm{~b}$, or vice-versa). After viewing the first two stimulus intervals, observers were asked to indicate, via a button press, whether the depth separation between the points was larger in the first or second interval. We used a method of constant stimuli with a standard stimulus presented in one interval (this could be either top-near or top-far), with a depth separation of $10 \mathrm{~cm}$ (22 min arc disparity, at the $1 \mathrm{~m}$ viewing distance, for an observer with interocular distance $6.5 \mathrm{~cm}$ : disparities were different for each observer, based on their different interocular distances), and a test stimulus with a depth chosen at random from one of this range: $1,4,7,10,13,16,19 \mathrm{~cm}$.

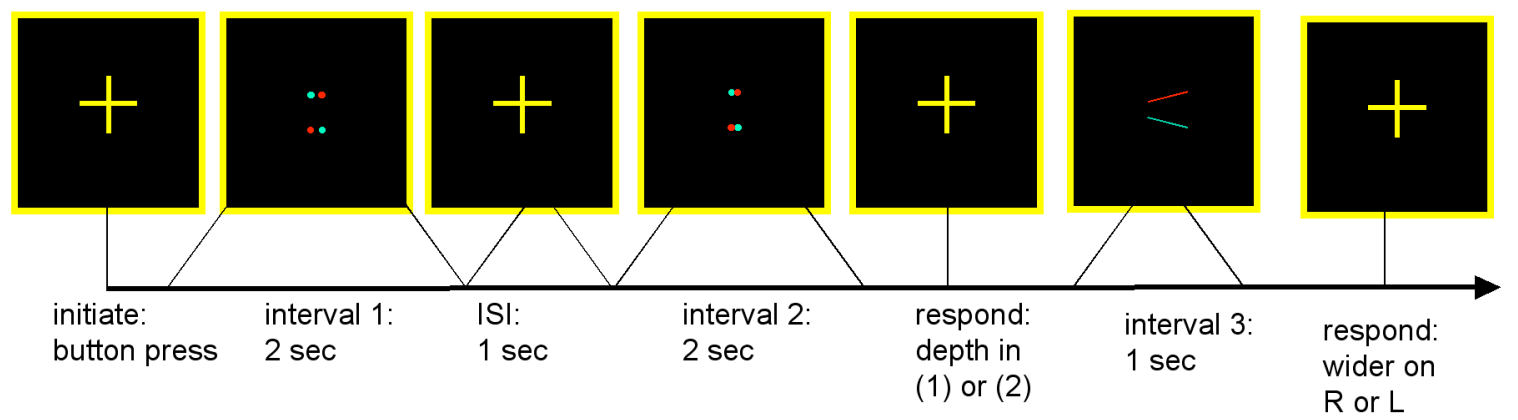

\section{Figure 2}

Illustration of the order of stimulus presentation and response intervals for the two tasks. 
Test and standard could each be top-near or top-far and randomly appeared in the first or second interval. Trials were run in blocks of 112, with the four different conditions randomly interleaved. In each block, 4 repetitions of each of the 7 depth intervals, in each of the 4 conditions, were presented. Three blocks of trials were completed by each observer, 12 trials were run per condition for each test depth.

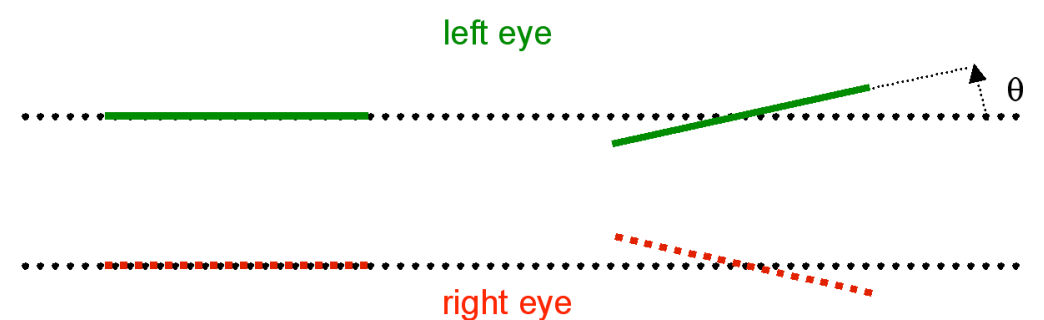

\section{Figure 3}

The stimulus for task 2. Green solid lines show stimulus to the left eye, right dotted lines show the stimulus to the right eye. Observers are asked if the nonius lines are wider apart on the right than the left. With zero cyclorotation the parallel configuration on the left should yield $50 \%$ 'left wider' responses.

After a further blank inter-stimulus interval (1 second) a third stimulus interval was presented, and observers asked to perform Task 2. Here we use horizontal nonius lines to psychophysically measure eye rotation (this is the preferred stimulus for measuring cyclovergence psychophysically, as suggested by Rogers \& Bradshaw, 1999, also see Howard, Ohmi \& Sun, 1993). If a pair of horizontal nonius lines is viewed, as shown in figure 3 , with the eyes correctly aligned, the image on each retina will also fall along horizontal meridians and the lines will be perceived as parallel and horizontal. If the eyes are cyclorotated outwards, the meridians will be rotated such that horizontal lines appear to be oriented down-right in the left eye, and up-right in the right eye. The observer would see this stimulus not as horizontally 
aligned, but rather the lines would appear to be more widely separated on the left side, than on the right side. To appear horizontal for such an eye-rotation, the nonius lines would actually have to have the opposite offset (figure 3, right), more widely separated on the right than the left. By using a range of stimulus line rotations, it is possible to measure cyclorotation from psychophysical responses. We used a two-alternative, single interval task, in which observers were shown one of seven possible stimuli with offset angle, $\theta$ (figure 3 ) drawn from one of: -3, -

$2,-1,0,1,2,3$ deg (where a positive angle is consistent with the right side being more widely separated). Observers were asked to indicate whether the lines were more vertically separated on the right or the left ("right side wider?"). In preliminary analyses we found no effect of teststandard order. The point of subjective equality (where the lines appear horizontal) gives a psychophysical measure of the cyclovergence.

When the observer gave their response, the trial ended, the fixation cross appeared, and when ready, the observer initiated the next trial with a button press.

\section{Results}

\section{Task 1: Depth data analysis and results}

We collected data from 24 observers, plotting proportion test-depth-bigger as a function of test depth, for the four test:standard conditions ('same' configurations: top-near-top-near, top-fartop-far; 'different configurations': top-near-top-far, top-far-top-near). One of our aims was to estimate the size of the bias (the difference between the PSE's for the two conditions), if any, between the same and different configurations. To do this, we averaged responses for the two same configurations. To combine the different conditions, we needed to transform the data, because the test and standard have opposite configurations for the two conditions. We replotted the data as proportion of 'top-near-longer', as a function of top-near minus top-far depth. 
Now the two different curves should lie close to one another, and responses can be averaged for each stimulus value. The combined transformed data for each of the same and different conditions were plotted separately for each observer.

The disparity range we used was specifically set up to be well above depth discrimination threshold. Previous work has suggested depth pedestal thresholds around 1 min arc, for a 20 min arc depth step (Blakemore, 1970; Badcock \& Schor, 1985). Here, our pedestal was approximately $20 \mathrm{~min}$ arc for the standard stimulus. The test stimulus could have disparity as much as $18 \mathrm{~min}$ below that value (1cm depth, 2 min arc disparity), or $19 \mathrm{~min}$ above it $(19 \mathrm{~cm}, 39$ min arc disparity). We had hoped that this large range would allow as many observers as possible to deliver useful data. However, many observers delivered data where responses for some, or all, of the range were very close to chance. We therefore started our analysis by considering whether we had any evidence that observer performance was different from chance. Rather than relying on poor estimates of slope from a sigmoid curve fit, we kept our initial analysis simple, and conducted a $\chi^{2}$ test (considering each test value of depth separation as a depth category) comparing the data with an expectation of chance performance for all categories.

Only 8 of our 24 data sets passed this test for both same and different conditions, 9 data sets passed the test if we considered only the same condition, and 10 if we considered only the different condition. This was a very surprising result, given that our choice of stimuli was based on making the task as easy as possible, with the stimulus range spanning around ten times published thresholds for this kind of experiment. Our suspicion is that it is related to our using naïve and largely unpractised observers. We comment on this point further in the Discussion. 
Our next aim was to determine, for the eight reliable participants, whether their performance differed between the same and different conditions. Again, using the simplest analysis first, we performed a $\chi^{2}$ test, this time comparing the two conditions with each other, with the expectation of identical performance for the two conditions. We found 6 observers who delivered data with significant differences ( 6 d.f. for all, DR: $\chi^{2}>38, p<10^{-6}$, LS: $\chi^{2}>38, p<10^{-6}$, BT: $\left.\chi^{2}=26.5, p=0.0002, \mathrm{HN}: \chi^{2}=16, p=0.014, \mathrm{AC}: \chi^{2}>38, p<10^{-6}, \mathrm{CG}: \chi^{2}>38, p<10^{-6}\right)$. There appeared to be no evidence for any difference between the conditions for observers VD $\left(\chi^{2}(6)=5.49, p=0.48\right)$ and $\mathrm{HH}\left(\chi^{2}(6)=6.22, p=0.4\right)$.

For the eight reliable observers we also performed a standard psychophysical analysis. Data were fitted with a cumulative normal distribution to find the best-fit psychometric function (psignifit 3.0 toolbox, Fründ, Haenel \& Wichmann, 2011). Threshold was defined as half the difference between the depth delivering a $75 \%$ longer response, and the depth delivering a $25 \%$ longer response. This can be expressed as a Weber-fraction by dividing by the standard depth value of $10 \mathrm{~cm}$. 
(a)
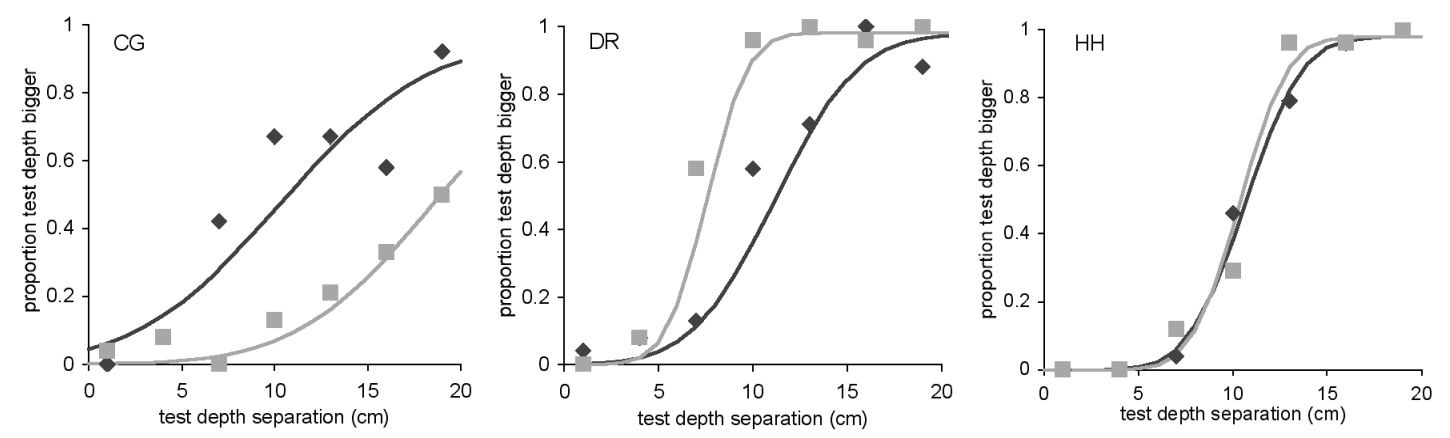

(b)

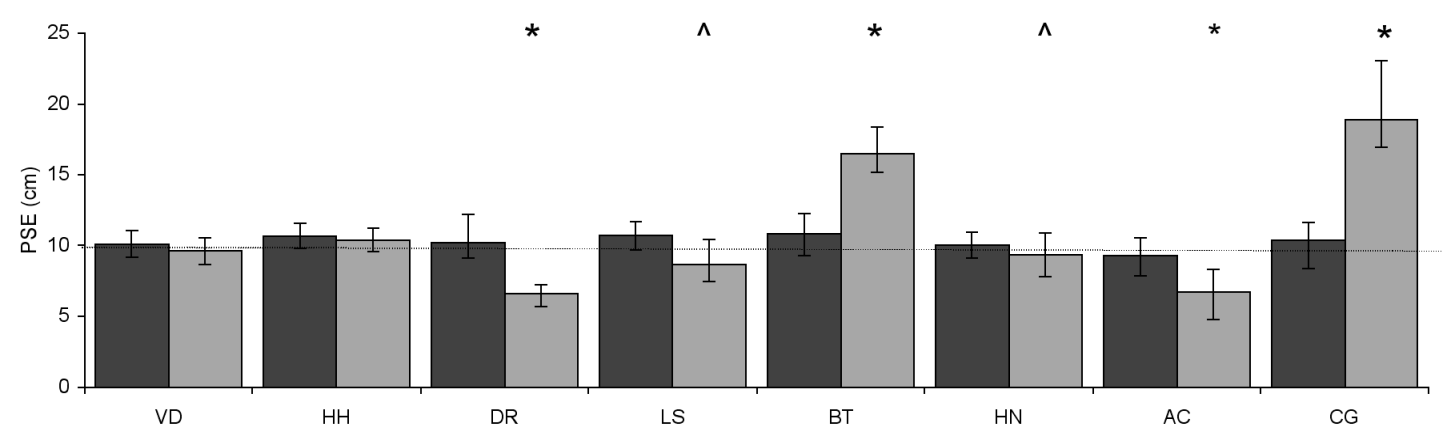

(c)

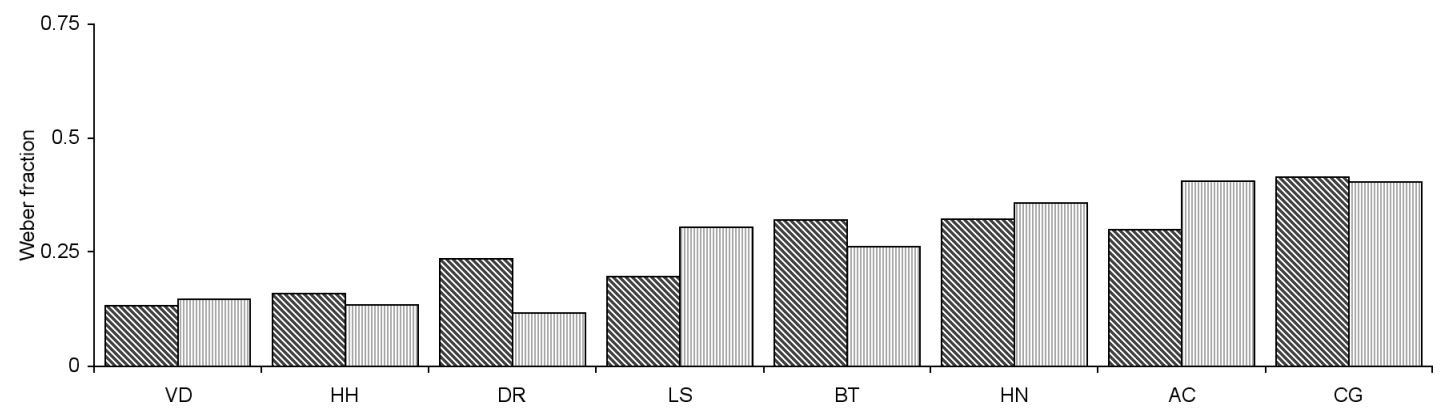

Figure 4

(a) Psychometric functions for individual observers for same (black diamond) and different (grey square) configurations. (b) PSE's for each observer, for each conditions (error bars are $95 \%$ confidence intervals; *: 95\% conf. intervals from PSE's do not overlap; ^: conditions significantly different according to chi-sq test.) (c) Thresholds shown as Weber fraction for each observer, for same (black diagonal stripe) and different (grey vertical stripe) conditions. 
Sample data sets from three of the eight reliable observers are shown in figure $4 a$. The offsets of the psychometric functions for the same and different conditions, demonstrate that there is a bias for some observers. Figure 4b shows PSE's for all 8 observers, for the two conditions, with error bars indicating 95\% confidence intervals for the fits (bootstrapped using 2000 throws per point, Wichmann \& Hill, 2001b). with starred bars showing observers for whom we can confidently say there is a bias (95\% confidence intervals on PSE's do not overlap) and bars with a hat $\left({ }^{\wedge}\right)$ showed those for whom the chi-squared test delivered significant differences. Figure 4c shows thresholds, expressed as Weber fractions, for the two conditions, for the 8 observers. Notice that although thresholds varied between observers, there was no systematic difference in sensitivity between the same and different conditions (paired t-test, $t(7)=0.56$, $p=0.62)$.

Bias was defined as the difference between the same PSE and the different PSE. A positive bias indicates that the top-near configuration was perceived as having a greater depth separation than the top-far configuration. Significance was tested based on the overlap of $95 \%$ confidence intervals. A significant difference in PSE, and thus a 'real', bias was evident for observers $\mathrm{DR}, \mathrm{AC}, \mathrm{BT}$ and $\mathrm{CG}$. For the remaining two who passed the $\chi^{2}$ test (LS, HN), this new analysis did not find a significant difference between conditions. All cumulative normal fits, apart from the one for CG, condition different, can be described as 'good' fits using the criterion that the observed deviances were within the $95 \%$ confidence bounds that we would expect based on the fitted function (Wichmann \& Hill, 2001a). 


\section{Modelling incorrectly interpreted viewing geometry or eye movements}

As discussed in the Introduction, we would expect systematic biases in the perceived depth of same and different conditions, if either viewing geometry or eye movements were not correctly interpreted by the visual system.

To extract binocular disparity correctly, the eyes must be in correspondence. This means that the axes of the two eyes must be aligned, or the viewing geometry known, including the axis around which torsional eye movements occur. In the Appendix, we describe in detail how the effects of a torsional eye movement could affect the depth perceived. If each of the eyes were subject to an outward cyclorotation of $\theta$ degrees, the difference in depth between different and same conditions would be given by:

$Z_{\text {diff }} \approx 4 D Y \sin \theta / I$,

where $Y$ is the vertical separation between the points in the 3-D world (in metres), $I$ is the interocular separation, and $\mathrm{D}$ is the viewing distance to the fixation point.

Slightly different predictions are obtained if the eyes remained aligned, but we consider depth perception errors occurring due to the tilt of the vertical horopter. That is, perceived depth might be determined by disparity relative to the tilted empirical vertical horopoter. We know that the empirical vertical horopter is inclined, (e.g. Siderov et al, 1999; Ledgeway \& Rogers, 1999) and that the inclination is consistent with the vertical meridians in the left and right eyes being slightly extorted (in other words, the vertical meridians are rotated outwards). This does not have exactly the same effect on the effective disparities as a cyclorotation, because only the vertical meridians are rotated, the horizontal meridians remain aligned. However, the effect is 
similar. Effective horizontal disparities for the top-far configuration will be smaller than for the top-near configuration, resulting in a bias between the two perceived depths. Following a similar logic to that above, an equation can be derived that links the depth difference between the two configurations with the vertical meridian extortion (where $\theta$ is the rotation, in opposite directions, of each eye's meridian, and the other symbols are as above):

$Z_{\text {diff }} \approx 4 D Y \tan \theta / I$,

Several different studies have demonstrated a tilt in the empirical vertical horopter. On the whole, studies agree that the extortion of the vertical meridians is around 1-2 deg (e.g. Siderov et al, 1999; Ledgeway \& Rogers, 1999, Schreiber et al., 2008). If we take a value of 2 deg, that gives an angle $\theta$ of $1 \mathrm{deg}$ (from equation 2). This would deliver a difference in depth of $5.8 \mathrm{~cm}$. Thus, we expect a positive bias at or near $5.8 \mathrm{~cm}$, if the vertical horopter tilt were responsible for the bias. Although there are individual differences in tilt reported in the literature (Grove et al, 2001), all observers show a backwards tilt. Because there was no consistent positive bias across participants in our experiments (figure 5c), we conclude that the tilt of the empirical vertical horopter cannot account for our data. 
(a)
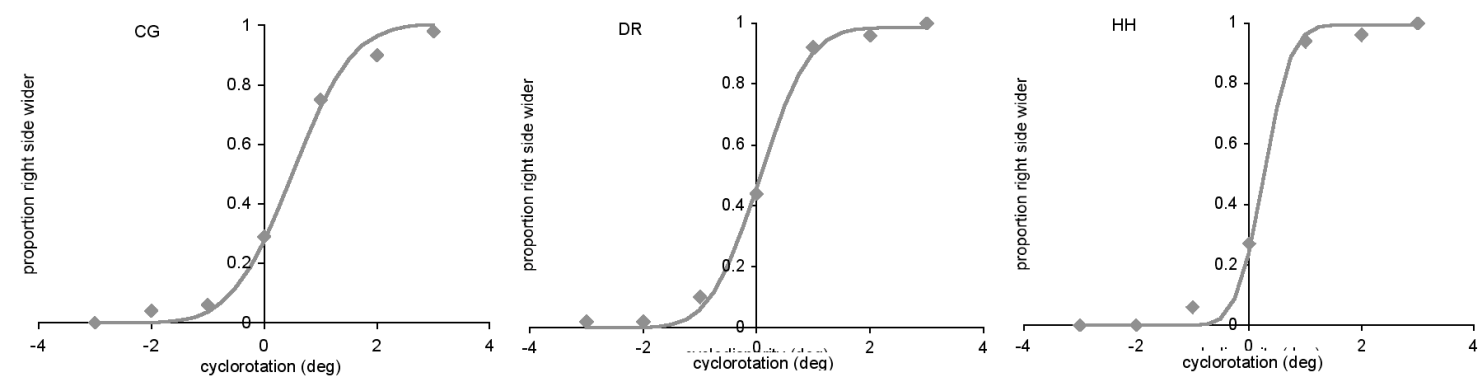

(b)

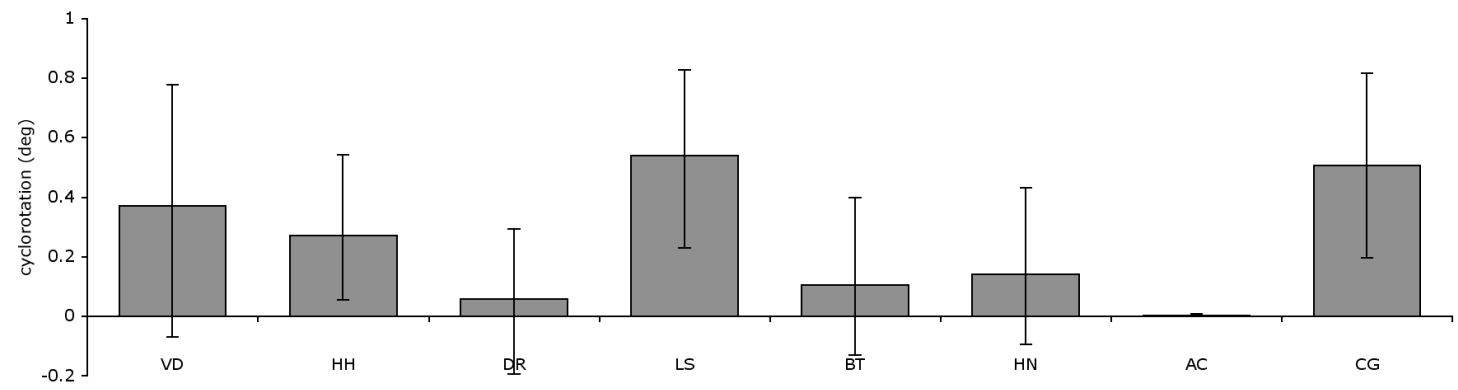

(c)

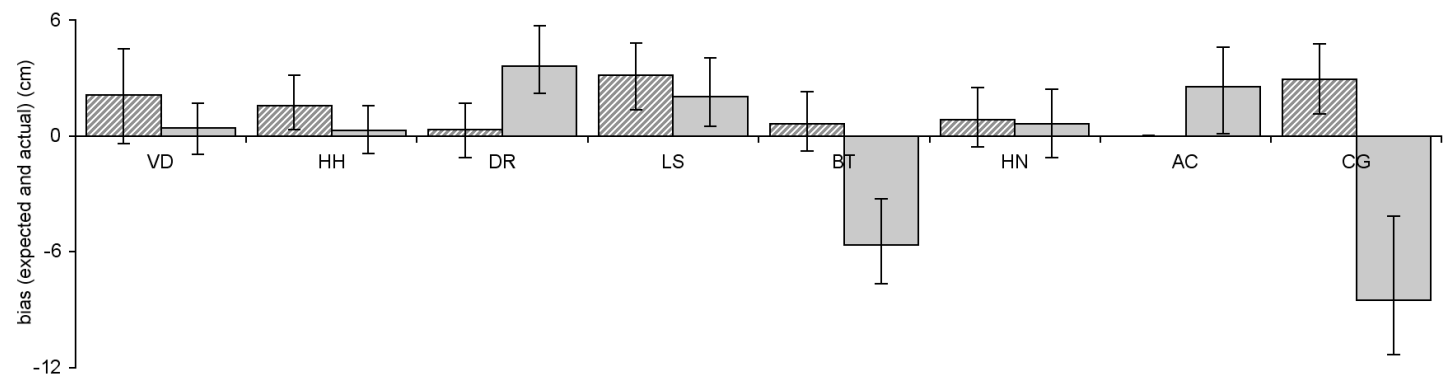

Figure 5

(a) Individual observer data for task 2, cyclovergence. (b) Summary data showing cyclovergence: the $50 \%$ point on the psychometric function. Error bars show $95 \%$ confidence intervals. (c) The expected bias, as calculated using equation 1 (striped bars, on left), based on cyclovergence data from (b), compared with measured bias (from figure $4 \mathrm{~d}$, light grey bars, on right). Error bars calculated from $95 \%$ confidence intervals. 


\section{Comparing bias and cyclovergence data (task 2)}

The cyclovergence data were gathered as part of the same experiment as the depth data. We collected proportion of 'right side wider' responses as a function of the rotation applied to the horizontal nonius line pair (figure 3). Data were fit with a cumulative normal to find the bestfitting psychometric function. Sample data from three observers are displayed in figure $5 a$. A measure of cyclovergence for each eye was given by the PSE of the fitted curve. Positive values are consistent with outward cylcorotation. Figure 5b shows estimates of cyclorotation for each of the eight observers. The average rotation was 0.17 deg and was significantly different from zero (two-tailed paired t-test, $t(7)=0.98, p=0.026$ ). In principle, this measured rotation could be due to two things (a) a fixed amount of rotation for an observer, regardless of the stimulus, or (b) rotation that is induced by each stimulus. This latter possibility is unlikely, because the stimuli were small, and previous research suggests that stimuli need to be quite large to induce cyclovergence. For example, many studies of cyclovergence use stimuli as large as 50 deg (e.g. Kertesz \& Sullivan, 1978) or even 75 deg (Howard \& Zacher, 1991). Howard (2002) suggests that stimuli should be at least 25 deg diameter to induce cyclovergence.

We used equation 1 to calculate the expected bias that should have resulted in the depth task, were the bias based entirely on an individual's cyclotorsion. Figure $5 c$ shows expected biases for each of the eight observers (diagonal striped bars, on left), compared with the biases obtained from the depth experiment (light vertical striped bars, on right bars). Expected and actual biases appear unrelated for each observer. There was no significant correlation between the two $(r=0.37, p=0.19)$, suggesting that there is no relationship between bias, as measured in the depth experiment, and cyclorotation, as measured psychophysically in concurrent trials. 
The logic of the cyclorotation experiment is that observers might hold a constant cyclorotation (called a cyclophoria). It is also possible that their eyes might rotate in response to the stimuli themselves. Given the small size of the stimuli used here, and the fact that a large stimulus is generally needed to drive cyclorotation, we considered this possibility unlikely. Nevertheless, the hypothesis can be checked by separately analysing cyclorotation data when the second depth interval contained a top-far stimulus, and when it contained a top-near stimulus. We collected proportion 'right side wider' responses as a function of the cyclorotation, and fit separate psychometric functions to the two data sets. Sample data sets for three observers are plotted in figure 6a. Top-near data are shown in black and top-far in grey. These three observers are representative, in that for most observers very little difference was seen between the two conditions. Cyclorotation was obtained by measuring PSE's for each data set, for each observer, and are plotted in figure 6b. Cyclorotations were very similar for the two conditions, and there was no significant difference between the two sets of data (paired t-test, $t(7)=0.79$, $p=0.28)$. 
(a)
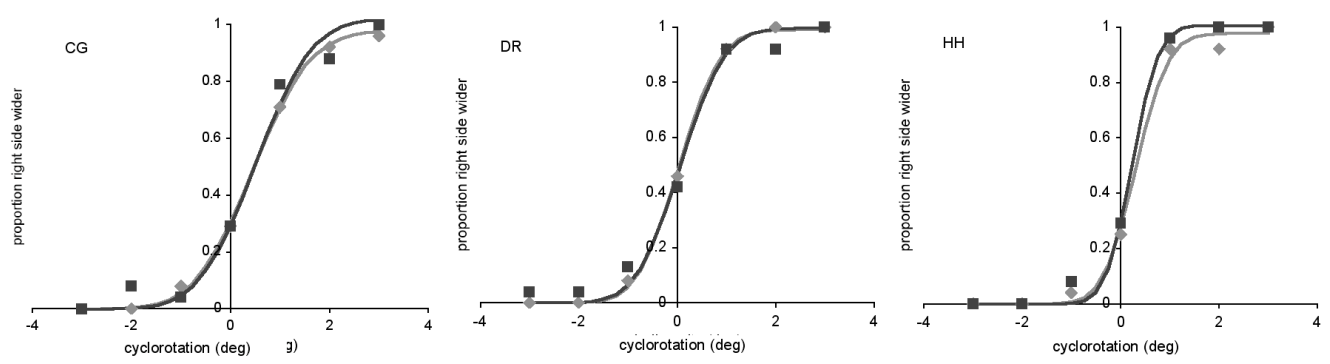

(b)

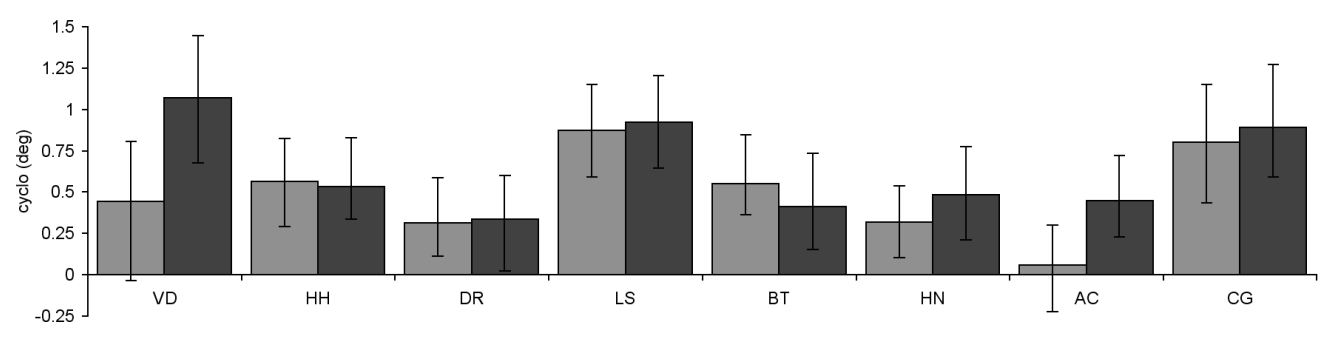

\section{Figure 6}

(a) Results for task 2, split by configuration: top-near (black) and top-far (grey). (b) Summary data for all observers showing the PSE on each psychometric function. There was no systematic difference between conditions. Error bars show 95\% confidence intervals.

\section{Discussion}

In this paper we explored a simple depth-interval judgement task that could, in principle, be performed accurately based on knowledge of the binocular disparities between pairs of points, and the viewing geometry. Many observers (16/24) were very poor at this task. Large, idiosyncratic, biases in perceived depth interval were found between the same and different conditions for those observers who were reliable. The biases did not appear to fall in a 
systematic direction, across the 8 reliable observers, nor were they of any specific size. In the sections below we assess whether the issues identified in the Introduction are responsible for the bias, and the extent to which there is evidence demonstrating biases from other work in the literature.

\section{Poor performance for many observers}

Data from 8/24 observers were investigated in detail. The other 16 produced data where we could not be sure that performance was different from chance for either the straightforward same condition or the different condition, or both. This result may appear striking on a casual inspection of the literature on depth from binocular disparity. Typically, $15-20 \%$ of observers in binocular vision experiments are rejected due to poor performance (report from an informal survey via the cvnet email group; archived at cvnet or available on request). Most psychophysical studies use tiny numbers of expert observers. Studies on binocular vision that have used large numbers of observers have found high proportions of participants who deliver poor performance. This occurs in other labs, even for what should be relatively easy depthrelated tasks (Wilmer, 2008; Hokoda, 1985, Porcar \& Martinez-Palomar, 1997, Watanabe et al, 2008), as well as our own lab where we have explored motion in depth (Nefs, O'Hare \& Harris, 2010). Many studies do not report in detail how their observers were chosen, nor how many were excluded. Our strategy here was to collect data from a sample of a normal young adult population (most were students), largely without specific training or experience, and to investigate in detail those whose performance was precise enough to model using standard psychophysical analyses. This allowed us to study the most reliable individuals in detail, while obtaining an estimate of how hard the task was for the population at large.

In a previous study, we tested a large number (62) of observers on a series of motion in depth tasks and a control dot-density task (Nefs et al, 2010). The idea of this latter task was to test 
whether some of our naïve observers were poorly motivated. If so we expected poor performance on all tasks. Although $50 \%$ of observers were poor at the motion in depth task, all delivered high sensitivity for the dot-density task. Our naïve observers appear well motivated, and are paid expenses for their time. These results demonstrated that they were well motivated, but that many people find tasks involving binocular disparity particularly difficult. We have no reason to believe that the observers studied here were any less well motivated than those used by Nefs et al (2010). We would like to speculate that the large proportion of poor observers may reflect something special about tasks involving binocular disparity: that considerable practise is required, compared with simpler visual judgments.

The depth task was clearly hard. All observers passed the TNO stereoacuity task, yet when asked to use their ability to discriminate one disparity step from a slightly larger or smaller one, they were unable to do so. We believe that several factors contribute to this. First, we chose a task that would be more difficult than stereoacuity. Depth separation discrimination is such a task, as has been described before (eg. Norman \& Todd, 1998; Blakemore, 1970). Second, the task was probably made even harder by using a 2-interval design, but this was essential, as we wanted to ensure observers actually needed to extract the depth increment to perform the task. Specifically, we used a task in which, for half of the trials, observers were required to compare depth intervals between points in different configurations. This means that they would not have been able to rely on a simple strategy, such as comparing the absolute disparities of points across intervals.

Although our task was chosen to be more challenging than a stereoacuity task, we did take care to ensure that our stimuli were within a range that was 'doable' according to the literature.

First, we used a combination of disparity between the two points, and vertical angular separation, that ensured the stimuli should be within Panum's fusional area. The disparity 
gradient was always well below the recognised fusion limit of 1 (Burt and Julesz, 1980; for the smallest, $1 \mathrm{~cm}$, depth separation the disparity gradient was 0.026 and for the largest, $19 \mathrm{~cm}$ separation it was 0.49 ). Second, our standard depth separation of $10 \mathrm{~cm}$ (22 min arc) should deliver a depth discrimination threshold of around $1.7 \mathrm{~min}$ arc, or $0.75 \mathrm{~cm}$, (Badcock \& Schor, 1985), well below the values of depth separations we used for the comparison stimuli. However, the extant literature could be misleading in terms of what we expect observers to achieve, because these classic studies used just a handful of expert observers.

One point that is worthy of note is that Howard \& Kaneko (1994) explored how cyclorotation of left and right eye images, in opposite directions, does not always result in apparent inclination, despite this manipulation adding the appropriate disparities to deliver inclination. Our stimuli are not unlike some of theirs: we added horizontal disparity between points, while they added cyclorotation. Further, their behavioural results were also inconsistent with measured cyclovergence of the eyes.

The 8 reliable observers delivered thresholds (defined as half the difference between $75 \%$ and $25 \%$ on the fitted psychometric functions) ranging from 1.3 to $4 \mathrm{~cm}$. Thus, our best observers' thresholds were around double those found by Badcock and Schor (1985). Given that they used simultaneous test-standard presentation, and we used sequential presentation, our best thresholds are as good as one would expect. Four of our reliable observers were experienced members of the lab (two postdocs, a PhD student, and one of the authors) who were naïve to the purposed of the experiment, but who had taken part in several (at least) previous studies using binocular disparity. In addition, in the group of 8 reliable observers, 4 were completely naïve, untrained, and unfamiliar with psychophysical experiments. This gave us confidence that there was not some aspect of the display (dot size, contrast) or apparatus (stereoscope alignment and arrangement) that was lacking in our experiments, compared to those of others. 
If there had been such a problem, the experiment should have been very difficult for all but the most experienced observers and would likely have delivered systematic biases. In sum, we consider that our small proportion of reliable observers genuinely reflects a population of 'normal' observers, who have not been specially chosen for the study.

\section{Binocular disparity relative to the horopter}

One way to define binocular disparity is that a non-zero disparity occurs whenever a point in space lies off the horopter. The issue of which points are on, or off, the horopter is complicated by the fact that the empirical horopter is different from the theoretical horopter. In particular, the empirical vertical horopter is known to be 'tipped back' slightly, the extent of the declination varying with viewing distance (Helmholtz, 1962; Cogan, 1979; Siderov, Hawerth and Bedell, 1999, Schreiber et al, 2008).

It is not clear whether the difference between empirical and theoretical horopters has consequences for depth perception. The empirical horopter is usually measured via alignment: observers are presented with a vertical line in one eye's view and asked to rotate a line in the other eye's view until aligned (Helmholtz, 1962). Alternatively a 'criterion of identical visual direction' can be used: points are alternately flashed in one eye, then the other, and the location of one is adjusted until there is no apparent motion between them (Nakayama, 1977, Ledgway \& Rogers, 1999). One study has compared the vertical horopter as measured by identical visual direction, with a vertical horopter as measured by the maximal sensitivity to binocular disparity, and found similar results (Siderov, Harwerth \& Bedell, 1999).

It is also not obvious how a tilted vertical horopter should affect depth interval judgements. On the one hand, the visual system might measure the separation between the images of the two scene points, with respect to the location of the vertical meridian in each eye. One would then 
expect all observers to show a systematic error, in the same direction, consistent with the backward tilt causing the 'effective' disparity to vary (see Appendix). On the other hand, one could argue that the visual system is cross-calibrated with other sensory information, such as from haptics, and may have a built-in mechanism to compensate for the horopter tilt.

The biases found in our experiments were not systematic across observers and certainly cannot be accounted for by the vertical horopter tilt alone. If there is a systematic bias, it is hidden by a much larger, idiosyncratic bias, which must be due to other causes.

\section{Viewing geometry}

Another potential cause of perceived depth biases could be a failure of the visual system to compensate for the relative orientations of the eyes. For example, if the eyes undergo cyclovergence, as can happen for fixation at different viewing distances, biases in depth perception could occur if disparity were measured with respect to vertical and horizontal meridians that are assumed to be aligned between the two eyes. This issue was described in detail earlier, and is shown diagrammatically in figure 7 . We psychophysically measured cyclorotation, during the same experiment in which depth interval judgements were made, but found no evidence to suggest that the former was linked to the latter (figure 5).

Observers viewed each stimulus for 2 seconds, partly because we found that shorter stimulus durations resulted in even poorer performance. Its is possible that observers made different patterns of eye rotations during that 2 second interval. The cyclorotation measure above would not pick this up, because it only measured final rotation position at the end of a trial. A reference-frame change due to different patterns of eye movements during viewing of the 'same' and 'different' stimuli could be a potential explanation of our results. Detailed measurement of this is beyond the scope of the current manuscript. 


\section{Other explanations?}

So far we have considered how disparity information is obtained by measuring distances between locations on the retina. If the visual system processed depth in this way, we could potentially obtain a depth map, detailing a disparity for every location in a scene, relative to fixation. If our observers had access to this sort of information, then we would have expected our discrimination task to be a very easy one for them to solve. For both the same and different conditions, they would merely have had to compare the magnitude of the disparity, while ignoring its sign. There is some evidence to suggest that this kind of representation is not used. Instead, it has been suggested that disparity is measured locally, relative to a local reference frame, determined by the surface on which a point lies (Petrov \& Glennerster, 2006; Glennerster \& McKee, 2004).

Consider our simplest condition, same, where for example, a top-far configuration was compared with another top-far configuration. Using a local reference frame should be straightforward, because one could imagine a reference frame based on the configuration in the first interval. The task would be to compare the stimulus in the second interval against that frame. For example, observers might choose to fixate the lower target (also the nearest). Essentially, observers could, at least in principle, compare the disparity between the two lower targets across the two intervals. Or, the disparity of the upper point could be measured with respect to fixation. The task would be reduced to 'which interval contained the largest uncrossed disparity with respect to fixation?'. It is unlikely that observers adopted that easier 'use disparity' strategy only for the 'same' condition, because we used a non-blocked design: same and different conditions were randomly interleaved, discouraging observers from developing specific strategies. 
The different condition needs additional information for the disparity to be processed without bias. For example, if observers chose to fixate on the lower target the task would require comparing opposite disparity sign steps, but for slightly different viewing distances (in one interval the lower target is the nearer, in the other, it is farther). Hence, an estimate of viewing distance (alternatively an estimate of eye position) is required to estimate metric depth without bias. Or, if observers chose to fixate on the nearest target, the task would require comparing the same sign disparity steps, but at different locations (above versus below fixation). This requires some knowledge of the shape of the vertical horopter to successfully convert to an estimate of metric depth.

If observers were simply responding to disparity, and not attempting to extract metric depth, we would then expect the same pattern of bias for all observers, because errors would be consistent with an assumption of fixed eye position and constant viewing distance. The idiosyncratic pattern of bias that we observed is not consistent with this interpretation. We would also expect a decreased sensitivity for the different condition compared to the same condition. However, we found no significant difference in sensitivity between those conditions, only a difference in PSE, reflecting the bias (figure $4 b$ and $4 c$ ). The consistent threshold results could indicate that the brain is attempting to extract metric depth for both tasks, and the idiosyncratic biases suggest that it is done incorrectly.

Comparing the relative depths between pairs of points, across two intervals in time, appears to be a very simple task, yet our results have revealed large biases and poor performance in general. If the issues of eye position and empirical vertical horopter do not account for the bias, then where does it come from? Almost all previous work on depth interval perception has explored thresholds, but not biases. It is well known that depth increment thresholds rise with increasing increment size (Ogle, 1953; Blakemore, 1970, Westheimer \& McKee, 1978). Depth 
interval discrimination for a pair of points is worse than depth order discrimination (Norman \& Todd, 1998). To our knowledge, no other studies have explicitly studied simple changes in configuration, for point-like objects, as we have done here.

One important point is that we used very sparse stimuli, delivering a minimum amount of information about the possible viewing distance or viewing geometry. It has previously been suggested that the disparity distribution present in a scene could, in principle, be used to obtain an estimate of viewing distance and hence reduce biases in perceived depth (Harris, 2004; Glennerster et al, 1998). O'Kane and Hibbard (2010) tested this suggestion but found no evidence for the visual system using more complex contextual information in this way. In their work, they found that adding a distribution of disparities into a surrounding pattern, specifically added to make the scene more ecologically valid, had no effect on the depth settings made. So far, then, there is no evidence that a more complex disparity-defined scene should deliver more consistent or more accurate performance. Of course, we do acknowledge that adding other sources of visual information should at some point, improve the perception of distance, and hence reduce bias. However, the purpose of this study was to specifically explore the simplest possible sparse scene.

Our stimuli, although very sparse, could in principle be interpreted as either a pair of points lying on a ground plane, or a pair of points lying on a ceiling plane. It is tempting to suggest that the visual system may treat these types of pattern differently, because ground-planes are ubiquitous in the world, and ceiling planes rarer. There is some evidence that the visual system can treat these differently. McCarley \& He (2000) used a visual search task to show that elements can be searched for more quickly when on a disparity-defined ground-plane than a ceiling plane. Bian and Anderson (2010) found an advantage in detecting changes to a ground-plane, or to objects on a ground-plane, in comparison to a ceiling plane. However, we 
found no systematic differences in sensitivity when comparing the two 'same' conditions, one of which is reminiscent of a ceiling plane and the other a ground plane. So, once again, our data cannot be explained by this suggestion.

\section{Summary and Conclusions}

In conclusion, our observers demonstrated remarkably poor performance in a depth interval judgement task, showing poor sensitivity to depth differences and large, idiosyncratic biases in perceived depth as a result of the configuration of points. These data cannot readily be accounted for by a consideration of possible failures to take account of the shape of the empirical horopter, the cyclorotation of the eyes, or the coordinate frame with which disparity is represented. Rather, they suggest that observers do not have ready access to accurate and reliable information about metric depth relations between points from binocular disparity and eye position alone. 


\section{References}

Badcock, D.R. \& Schor, C.M. (1985). Depth-increment detection function for individual spatial channels. Journal of the Optical Society of America. A2, 1211-1215.

Bian, Z. \& Anderson, G.J. (2010). The advantage of a ground surface in the representation of visual scenes. Journal of Vision, 10(8):6, 1-19.

Blakemore, C. (1970). The range and scope of binocular depth discrimination in man. Journal of Physiology, 211, 599-622.

Brainard, D. H. (1997). The Psychophysics Toolbox. Spatial Vision, 10:433-436.

Breitmeyer, B., Julesz, B., \& Kropfl, W. (1975). Dynamic random-dot stereograms reveal updown anisotropy and left-right isotropy between cortical hemifields. Science, 24, 269-270.

Brenner, E. \& Landy, M.S. (1999) Interaction between the perceived shape of two objects. Vision Research, 39, 3834-3848.

Burt, P. \& Julesz, B. (1980) A disparity gradient limit for binocular fusion. Science, 208, 615617.

Cogan, A.I. (1979). The relationship between the apparent vertical and the vertical horopter. Vision Research, 19, 655-665.

van Ee, R., \& van Dam, L.C.J. (2003). The influence of cyclovergence on unconstrained stereoscopic matching. Vision Research, 43, 307-319.

Freeman, T.C.A. (2001) Transducer models of head-centred motion perception. Vision Research, 41, 2741-2755.

Fründ, I., Haenel, N.V. \& Wichmann, F.A. (2011) Inference for psychometric functions in the presence of nonstationary behavior. Journal of Vision (in press).

Glennerster, A, \& McKee, S. (2004). Sensitivity to depth relief on slanted surfaces. Journal of Vision, 4. 378-387.

Glennerster, A., Rogers, B. J. \& Bradshaw, M. F. 1998 Cues to viewing distance for stereoscopic depth constancy. Perception 27, 1357-1366. 
Grove, P.M., Kaneko, H., \& Ono, H. (2001). The backward inclination of a surface defined by empirical corresponding points. Perception, 30, 411-429.

Harris, J.M. (2004) Binocular vision: moving closer to reality. Philosophical Transactions of the Royal Society, A. 362, 2721 - 2739.

Helmholtz, H. von (1867/1910/1962). Treatise on physiological optics, vol. III, trans. and ed. J. P. C. Southall. Dover. (Translated from the 3rd German edition, English edition 1962).

Hokoda, S. C. (1985). General binocular dysfunctions in an urban optometry clinic. Journal of the American Optometric Association, 56, 560-562.

Howard, I.P. (2002). Seeing in depth: Volume 1 Basic Mechanisms. Oxford University Press, New York.

Howard, I.P., \& Zacher, J.E. (1991). Human cyclovergence as a function of stimulus frequency and amplitude. Experimental Brain Research, 85, 445-450.

Howard, I.P., Ohmi, M., \& Sun, I. (1993). Cyclovergence - a comparison of objective and psychophysical measurements, Experimental Brain Research, 16, 349-355.

Howard, I. P., \& Kaneko, H. (1994). Relative shear disparities and the perception of surface inclination. Vision Research, 34, 2505-2517.

Johnston, E.B. (1991). Systematic distortions of shape from stereopsis. Vision Research, $31,1351-1360$.

Kertesz, A.E., \& Sullivan, M.J. (1978). The effect of stimulus size on human cyclofusional response. Vision Research, 18, 567-571.

Ledgeway, T., \& Rogers, B.J. (1999). The effects of eccentricity and vergence angle upon the relative tilt of corresponding vertical and horizontal meridian revealed using the minimum motion paradigm. Perception, 28, 143-153.

Manning, M.L., Finlay, D.C., Neill, R.A., \& Frost, B.G. (1987). Detection threshold differences to crossed and uncrossed disparities. Vision Research, 27, 1683-1686. 
Maxwell, J.S., \& Schor, C.M. (2006). The coordination of binocular eye movements: Vertical and torsional alignment. Vision Research, 46, 3537-3548.

McCarley, J.S., \& He, Z. (2001). Sequential priming of 3-D perceptual organization. Perception \& Psychophysics, 63, 195-208.

Mitsudo, H. (2007). Illusory depth induced by binocular torsional misalignment. Vision Research, 47, 1303-1314

Mitsudo, H., Kaneko, H., \& Nishida, S. (2009). Perceived depth of curved lines in the presence of cyclovergence. Vision Research, 49, 348-361.

Nakayama, K. (1977). Geometric and physiological aspects of depth perception. Proceedings of the Society of Photo-Optical Instruments of England, 120, 2-9.

Nefs, H. T., \& Harris, J. M. (2007). Vergence effects on the perception of motion in depth, Experimental Brain Research, 183, 313-322.

Nefs, H. T., \& Harris, J. M. (2008). Induced motion in depth and the effects of vergence eye movements. Journal of Vision, 8(3):8, 1-16

Nefs, H.T., O'Hare, L. \& Harris, J.M. (2010). Two independent mechanisms for motion-in-depth perception: evidence from individual differences. Frontiers in Psychology, 1:155. doi: 10.3389/fpsyg.2010.00155 Norman, J.F., \& Todd, J.T. (1998). Stereoscopic discrimination of interval and ordinal depth relations on smooth surfaces and in empty space. Perception, 27, 257-272

Ogle, K.N. (1953). Precision and validity of stereoscopic depth perception from double images. Journal of the Optical Society of America, 43, 907-913.

Ogle, K.N. (1958). Note on stereoscopic acuity and observation distance, Journal of the Optical Society of America, 48, 794-798.

O'Kane LM, \& Hibbard PB (2010) Contextual effects on perceived three-dimensional shape. Vision Research, 50, 1095-1100. 
Pelli, D. G. (1997). The VideoToolbox software for visual psychophysics: Transforming numbers into movies. Spatial Vision, 10:437-442.

Perrone, J.A., \& Krauzlis, R.J. (2008). Vector subtraction using visual and extraretinal motion signals: A new look at efference copy and corollary discharge theories. Journal of Vision, 8, $1-14$.

Petrov, Y., \& Glennerster, A. (2006). Disparity with respect to a local reference plane as a dominant cue for stereoscopic depth relief. Vision Research, 46, 4321-4332.

Previc, F.H., Breitmeyer, B.G., \& Weinstein, L.F. (1995). Discriminability of random-dot stereograms in three-dimensional space. International Journal of Neuroscience, 80, 247-53. van Rijn, L.J., Van Der Steen, J., \& Collewijn, H. (1992). Visually induced cycloversion and cyclovergence. Vision Research, 32, 1875-1883.

Porcar, E., and Martinez-Palomera, A. (1997). prevalence of general binocular dysfunction in a population of university students. Optom. Vision Sci. 74, 111-113.

Rogers, B.J., \& Bradshaw, M.F. (1999). Disparity minimisation, cyclovergence, and the validity of nonius lines as a technique for measuring torsional alignment. Perception, 28, 127-141.

Scarfe, P., \& Hibbard, P.B. (2006). Disparity-defined objects moving in depth do not elicit threedimensional shape constancy. Vision Research, 46, 1599-1610.

Schreiber, K. M., Hillis, J. M., Filippini, H. R., Schor, C. M., \& Banks, M. S. (2008). The surface of the empirical horopter. Journal of Vision, 8(3):7, 1-20

Siderov, J., Harwerth, R.S., \& Bedell, H.E. (1999). Stereopsis, cyclovergence and the backwards tilt of the vertical horopter. Vision Research, 39, 1347-1357.

Watanabe, Y., Kezuka, T., Harasawa, K., Usui, M., Yaguchi, H., \& Shioiri, S. (2008). A new method for assessing motion-in-depth perception in strabismic patients. British Journal of Ophthalmology, 92, 47-50.

Westheimer, G., \& McKee, S.P. (1978). Stereoscopic acuity for moving retinal images. Journal of the Optical Society of America, 68, 450-455. 
Wichmann, F.A., \& Hill, N.J. (2001a). The psychometric function: I. Fitting, sampling and goodness of fit. Perception and Psychophysics, 63(8), 1293-1313

Wichmann, F.A., \& Hill, N.J. (2001b). The psychometric function: II. Bootstrap-based

confidence intervals and sampling. Perception and Psychophysics, 63(8), 1314-1329.

Wilmer, J. B. (2008). How to use individual differences to isolate functional organization, biology, and utility of visual functions; with illustrative proposals for stereopsis. Spat. Vis. 21, $561-579$. 


\section{Appendix}

To extract binocular disparity correctly, the eyes must be in correspondence. This means that

(a)

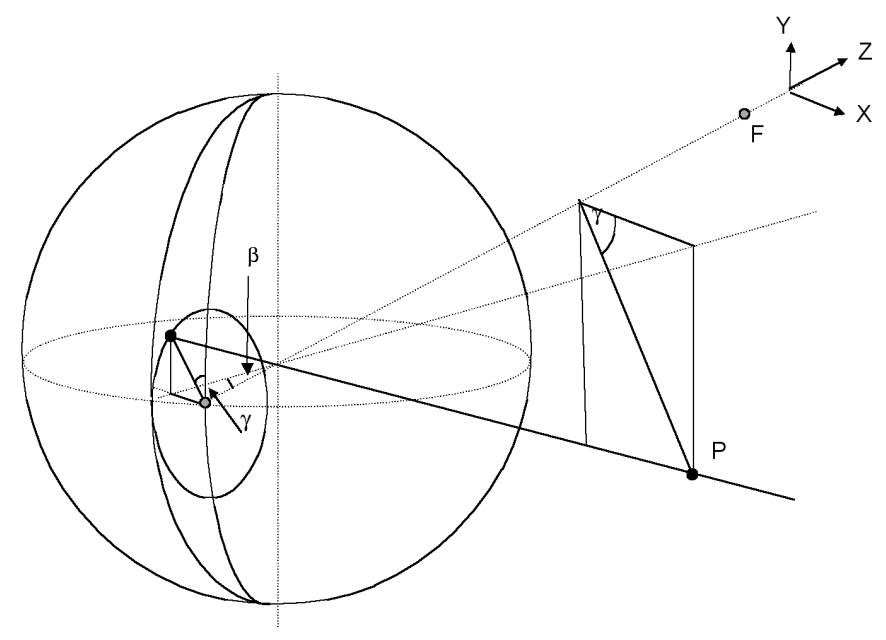

(b)
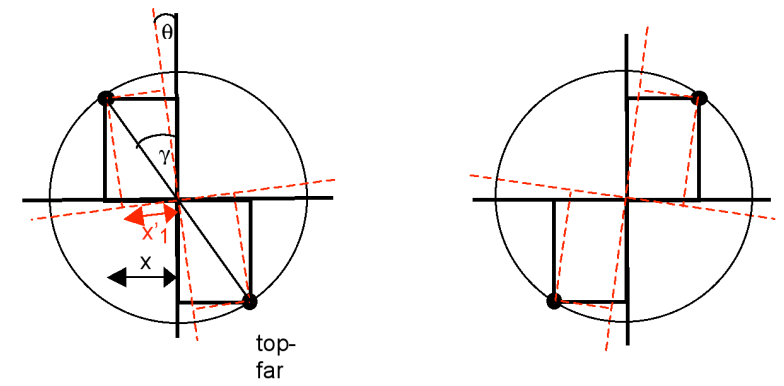

(c)
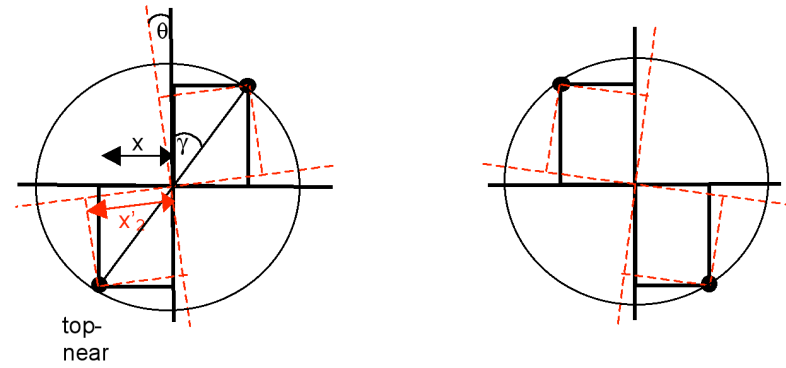

\section{Figure 7}

(a) Diagram showing points $\mathrm{P}$ (bottom-near point) and $\mathrm{F}$ in the world, and their projections onto the retina of the left eye. (b) is the view from behind the eye of the circular region shown in (a), on the left for the left eye and right for the right eye. Projections of both the top-far and bottom-near point are shown. The red dotted lines are the extorted meridians if the eyes experienced outward cyclorotation. Note the distance $x_{1}$ is smaller than distance $\mathrm{x}_{1}$, the horizontal offset of point $\mathrm{P}$ before cyclorotation. (c) Shows the opposite configuration, top-near. Notice now, for the same cyclorotation, that the distance $x_{2}$ is larger than distance $x_{1}$. 
the axes of the two eyes must be aligned, or the viewing geometry known, including the axis around which torsional eye movements occur. Figure 7 shows how the locations of image points, and hence their binocular disparity, can be affected by torsional eye movements.

In figure $7 \mathrm{a}$, the observer views a point, $\mathrm{P}$, located nearer and below the fixation point, $\mathrm{F}$. The projection of the point onto the retina is shown, and a circle forming a cross-section through the back of the retina, centred on the fixation point. Figure $7 \mathrm{~b}$ shows an expanded view of the cross-section through each eye, as viewed from behind the eye. The solid lines show the horizontal and vertical meridians.. This diagram shows the images of the lower-near point, $\mathrm{P}$ (above fixation in this projected image, as shown in figure 7a), and also the top-far point (below fixation in the projection). For simplicity we discuss the case where the eyes are fixated midway between the locations of the two points (fixation point F). These images correspond to a pair of point objects in the world, lying an equal distance above and below the horizontal mid-line. The lower object (upper object at the eye due to image inversion) is located nearer in depth than the midpoint (lower-near condition), and therefore positioned to the left in the left eye's projection, and the right in the right eye's projection, by an angular separation, $\beta$. This is represented by distance $x$, where $\sin \beta=x / r$ ( $r$ is the distance between the nodal point and the fovea), in figure $7 \mathrm{~b}$. The horizontal binocular disparity between that point and fixation is defined as $2 \beta$. The further object (lower point in image) is located further than the midpoint, by the same depth. If we assume the distance to fixation is much larger than the depths of points, its location is also offset by $\mathrm{x}$ in each eye, in the opposite direction. Its horizontal binocular disparity with respect to the midpoint is therefore $-2 \beta$. The relative disparity between the two points is therefore, $\delta=4 \beta$. 
Now consider what happens if the eyes undergo a slight cyclo-rotation outwards (shown by the red dashed lines). The horizontal offset of each point from the midpoint is now smaller, equal to $x_{1}{ }^{\prime}$, which is related to the original $x$ by:

$x_{1}^{\prime}=x \sin (\gamma-\theta) / \sin (\gamma)$

where $\gamma$ is the angle between the original vertical axis and a line joining the image of $P$ to the image of $F$, and $\theta$ is the cyclo-rotation angle of the eye. The apparent angular separation, $\beta$ ', is given by:

$\sin \left(\beta^{\prime}\right)=x_{1}{ }^{\prime} \sin (\beta) / x$

The effective binocular disparity (relative to the extorted horizontal-vertical axis) between the two points is therefore given by:

$\delta_{1}{ }^{\prime}=4 \sin ^{-1}[\sin (\beta) \sin (\gamma-\theta) / \sin (\gamma)]$

The same direction of cyclorotation has a different effect on objects arranged in the other configuration (top-near). Figure 7c shows the effects of cyclorotation when the upper object (lower in image) is near and the lower object (upper in image) far. Now, the horizontal offset of each point is larger than before the rotation, given by:

$x_{2}^{\prime}=x \sin (\gamma+\theta) / \sin (\gamma)$

And the effective disparity is correspondingly larger: 
$\delta_{2}^{\prime}=4 \sin ^{-1}[\sin (\beta) \sin (\gamma+\theta) / \sin (\gamma)]$

Thus, the apparent binocular disparity will be larger for the top-near configuration than for the top-far configuration. Note that the expressions derived in equations (A3) and (A5) are also obtained if the observer fixates either target. Using the above equations, is it possible to predict the expected depth bias, if observers were subject to a small cyclotorsion.

If we make some simplifying assumptions, that the viewing distance to the points is much larger than the depth separation between them, and that they are located close to, or at, the midline, the apparent depths can be calculated. Specifically, we assume (i) $\beta \approx \sin \beta$; and (ii) $\delta_{1}{ }^{\prime} \approx \sin$ $\delta_{1}{ }^{\prime}$, and $\delta_{2}{ }^{\prime} \approx \sin \delta_{2}{ }^{\prime}$, then from equations $A 3$ and $A 5$, the disparity difference between the two situations is simplified to:

$\delta_{2}{ }^{\prime}-\delta_{1}{ }^{\prime} \approx(4 \beta / \sin (\gamma))(\sin (\gamma+\theta)-\sin (\gamma-\theta))$

and using standard trigonometric identities this becomes:

$\delta_{2}{ }^{\prime}-\delta_{1}{ }^{\prime} \approx 8 \beta \sin \theta / \tan (\gamma)$

From above, we know that $\delta=4 \beta$, then equation $A 7$ is simplified to:

$\delta_{2}{ }^{\prime}-\delta_{1}{ }^{\prime} \approx 2 \delta \sin \theta /(\tan (\gamma))$.

From further trigonometry, we can estimate $\tan (\gamma)$ as:

$\tan (\gamma) \approx I Z / 2 Y D$ 
(a)
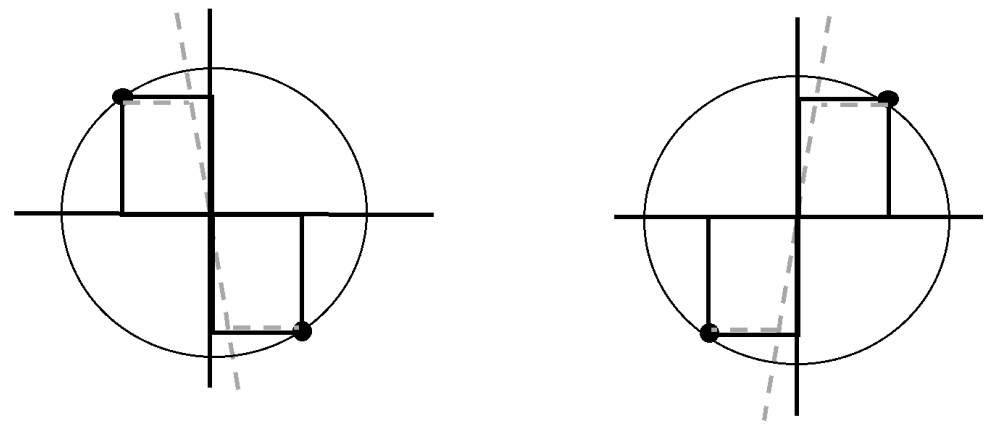

(b)
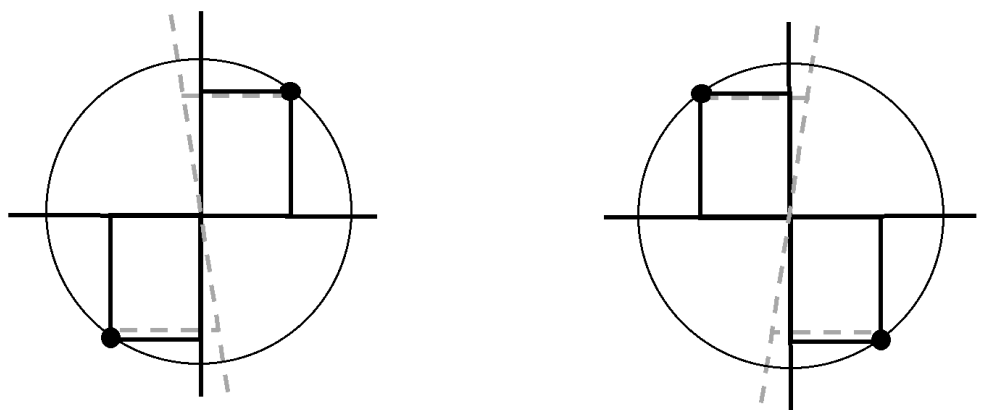

\section{Figure 8}

Diagram to illustrate the effects of extorting the vertical midlines only for the top-far (a) and top-near (b) configurations respectively.

The difference between effective $Z$ distances for top-near $Z_{2}{ }^{\prime}$, and top-far $Z_{1}{ }^{\prime}$ is:

$Z_{\text {diff }} \approx Z_{2}^{\prime}-Z_{1}^{\prime}$

And, substituting from $A 7$ and $A 9$ and using $Z \approx \delta D^{2} / \mathrm{l}$ :

$Z_{\text {diff }} \approx 4 D Y \sin \theta / I$,

Where $Y$ is the vertical separation between the points in the 3-D world (in metres), I is the interocular separation, and $D$ is the viewing distance to the fixation point. 
Slightly different predictions are obtained if the eyes remained aligned, but we consider depth perception errors occurring due to the tilt of the vertical horopter. Following a similar logic to that above, an equation can be derived that links the depth difference between the two configurations with the vertical meridian extortion (where $\theta$ is now the rotation, in opposite directions, of each eye's meridian, and the other symbols are as above):

Zdiff $\approx 4 \mathrm{D} Y \tan \theta / \mathrm{I}$

Equations $\mathrm{A} 7$ and $\mathrm{A} 8$ provide predictions for the expected depth difference, or bias, between the two stimulus configurations. 